\title{
The bifurcation of periodic orbits of one-dimensional maps
}

\author{
LOUIS BLOCK AND DAVID HART \\ Department of Mathematics, University of Florida, Gainesville, Florida 32611, USA
}

(Received 5 May 1982)

Abstract. The bifurcation of $C^{1}$-continuous families of maps of the interval or circle is studied. It is shown, for example, that period-tripling cannot occur. This yields topological properties of the stratification of $C^{1}(I, I)$ induced by the Sarkovskii order, and corresponding bifurcation properties.

\section{Introduction}

This paper deals with the bifurcation theory of maps of the interval and of the circle. We are interested in how the least period of a periodic point can change in a family which is continuous with respect to the $C^{1}$ topology. More precisely, if a map $f_{s}$ in a $C^{1}$-continuous family $\left\{f_{t}\right\}$ has a periodic point $x_{s}$ of period $k$ (which will always mean least period), we ask what may be the period of a periodic point $x_{t}$ (of $f_{t}$ ) near $x_{s}$, where $t$ is near $s$. If the period of $x_{t}$ is $2 k$, but $f_{s}$ has no $2 k$-periodic point near $x_{s}$, we say a period-doubling bifurcation occurs; if $3 k$, period-tripling, etc. Period-doubling bifurcations have been studied extensively (see for example [6], [7]). The first theorem of this paper implies that for $C^{1}$-continuous families of maps of compact one-dimensional spaces, period-tripling, quadrupling, etc., bifurcations do not occur. This is easily seen to be false for families which are only $C^{0}$-continuous.

THEOREM 1. Suppose $\left(f_{n}\right)$ is a sequence of maps in $C^{1}(I, I)$ or $C^{1}\left(S^{1}, S^{1}\right)$ and $\left(f_{n}\right)$ converges to $f$ (in $C^{1}(I, I)$ or $C^{1}\left(S^{1}, S^{1}\right)$ ). Suppose each $f_{n}$ has a periodic point $x_{n}$ of period $k$ (where $k$ is a fixed positive integer). Suppose some subsequence of $\left(x_{n}\right)$ converges to $x$. If $k$ is odd, $x$ is a periodic point of $f$ of period $k$. If $k$ is even, $x$ is a periodic point of $f$ of period $k$ or $\frac{1}{2} k$.

Using theorem 1, we show that the Sarkovskii ordering provides a stratification of $C^{1}(I, I)$ and gives topological restrictions on one parameter families in $C^{1}(I, I)$. Let $F(n)$ denote the set of maps in $C^{1}(I, I)$ which have a periodic point of period $n$. We will use the symbol $\triangleleft$ to denote the Sarkovskii order,

$$
2^{n} \triangleleft 2^{n+1} \triangleleft \cdots \triangleleft 2^{n+1} \cdot 5 \Delta 2^{n+1} \cdot 3 \triangleleft \cdots 2^{n} \cdot 5 \triangleleft 2^{n} \cdot 3 \text {. }
$$

Sarkovskii showed that if $n \triangleleft m$ then $F(m) \subseteq F(n)([5],[10],[12])$. Let $G(n)$ denote those $f \in F(n)$ such that $f \notin F(m)$ if $n \Delta m$. We may include the symbol $\infty$ by defining $k \triangleleft \infty$ if $k=2^{i}$, for some integer $j \geq 0$, and $\infty \triangleleft k$, otherwise. Those maps in $F(\infty)$, but not $F(m)$ for any $m$ with $\infty \triangleleft m$, will be denoted $G(\infty)$. 
The following corollaries follow immediately from theorem 1 and Sarkovskii's theorem. The second corollary was known to Misiurewicz [8].

CoROllaRy 1. If $n$ is not a power of $2, F(n)$ is a closed set.

COROLlaRY 2. If $n$ is a power of 2 , the closure of $F(n)$ is contained in $F\left(\frac{1}{2} n\right)$.

It follows at once from corollary 2 that $F(\infty)$ is closed. Hence the closure of the set of maps in $C^{1}(I, I)$ with positive topological entropy is contained in $F(\infty)$. The following result characterizes $F(\infty)$ as those maps whose set of periodic points is not closed.

THEOREM 2. Let $f \in C^{1}(I, I)$ or $f \in C^{1}\left(S^{1}, S^{1}\right)$ and suppose the set of periodic points of $f$ is a closed set. Then $f$ has only finitely many periods.

In [11], Nitecki gives an example of a continuous $f \in G(\infty)$ whose periodic points are closed; the above theorem shows this is a pathology of $C^{0}(I, I)$. Also, theorem 2 and [11] imply that for any $f \in G(\infty)$ which is $C^{1}$, there is a non-wandering point whose (infinite) orbit is 'separated to all orders.' If $f \in G(\infty)$ is $C^{3}$ and has negative Schwarzian derivative, the orbit structure of $f$ is completely described by Misiurewicz in [8] under the assumption that $f$ is unimodal.

Our final result uses theorem 1 and the stability theorem of [1] to obtain an intermediate value result for families in $C^{1}(I, I)$, in terms of the Sarkovskii ordering.

THEOREM 3. Let $f_{s}$ be a continuous arc in $C^{1}(I, I), 0 \leq s \leq 1$, with $f_{0} \in G(n)$ and $f_{1} \in G(m)$. Then for any $k$ such that $n \triangleleft k \triangleleft m$, there exists $s_{k}$ such that $f_{s_{k}} \in G(k)$, and $i<j$ implies $s_{i}<s_{j}$.

Note that $n, m$ or $k$ may be $\infty$. It follows that any such arc with $h\left(f_{0}\right)=0$ but $h\left(f_{1}\right)>0$, where $h$ denotes entropy, must pass through $G(\infty)$. Theorems 2 and 3 make the following strengthening of the remarks after corollary 2 seem reasonable.

CONJECTURE. The closure of the maps in $C^{1}(I, I)$ which have positive entropy is exactly $F(\infty)$. The boundary of that set, and of the set of maps in $C^{1}(I, I)$ with only finitely many periods, is exactly $G(\infty)$.

Of course, the above results do not exhaust the bifurcation behaviour of interval maps. The 'particle-antiparticle' bifurcation (which is sometimes called 'saddlenode' in higher dimensions) which occurs as a segment of the graph of $f$ (or of $f^{k}$ ) moves across the diagonal is the most obvious additional phenomenon. The cluster of bifurcations which occurs as $f_{s}$ moves into the positive-entropy region which results in homoclinic orbits is another (which we shall address in another paper).

\section{Proof of theorem 1}

We begin the proof of theorem 1 by proving two lemmas.

LEMMA 1. Let $\left\{p_{1}, p_{2}, \ldots, p_{k}\right\}$ be a periodic orbit of $f \in C^{1}(I, I)$ of period $k$ where $k>2$ and $p_{1}<p_{2}<\cdots<p_{k}$. There are points $y$ and $z$ in the interval $\left[p_{1}, p_{k}\right]$ with $f^{\prime}(y)>0$ and $f^{\prime}(z) \leq-1$. 
Proof. For some integer $m$ with $1<m<k$, either $f\left(p_{m}\right)=p_{1}$, or $f\left(p_{m}\right)=p_{k}$. If $f\left(p_{m}\right)=p_{1}$, there is a point $y \in\left[p_{m}, p_{m+1}\right]$ with $f^{\prime}(y)>0$. If $f\left(p_{m}\right)=p_{k}$, there is a point $y \in\left[p_{m-1}, p_{m}\right]$ with $f^{\prime}(y)>0$.

Now, let $i$ be the smallest element of $\{1,2, \ldots, k\}$ with $f\left(p_{i}\right)<p_{i}$. Then $i>1$, $f\left(p_{i}\right) \leq p_{i-1}$, and $f\left(p_{i-1}\right) \geq p_{i}$. Hence by the Mean Value Theorem, there is a point $z \in\left(p_{i-1}, p_{i}\right)$ with $f^{\prime}(z) \leq-1$.

Lemma 2. Suppose $\left(f_{n}\right)$ converges to $f$ (in $C^{1}(I, I)$ or $\left.C^{1}\left(S^{1}, S^{1}\right)\right)$ and suppose that for each $n, x_{n}$ is a periodic point of $f_{n}$ of period $k$, where $k$ is a fixed positive integer with $k>2$. If $\left(x_{n}\right)$ converges to $x$ then $x$ is a fixed point of $f^{k}$ but not a fixed point of $f$.

Proof. By continuity, $x$ is a fixed point of $f^{k}$, so it suffices to prove that $x$ is not a fixed point of $f$. We assume that $x$ is a fixed point of $f$ and obtain a contradiction.

First suppose that $\left(f_{n}\right)$ converges to $f$ in $C^{1}(I, I)$. Let $p_{n}$ denote the smallest element and $q_{n}$ the largest element of the orbit of $x_{n}$. By taking subsequences, we can assume that $\left(p_{n}\right)$ converges to $p$ and $\left(q_{n}\right)$ converges to $q$. There are positive integers $i$ and $j$ such that

$$
\left(f_{n}\right)^{i}\left(x_{n}\right)=p_{n} \quad \text { and } \quad\left(f_{n}\right)^{j}\left(x_{n}\right)=q_{n}
$$

for infinitely many $n$. Hence, by continuity,

$$
f^{i}(x)=p \quad \text { and } \quad f^{j}(x)=q
$$

Since $f(x)=x$, we have $x=p=q$. By lemma $1, f^{\prime}(x) \geq 0$ and $f^{\prime}(x) \leq-1$, a contradiction.

Now suppose that $\left(f_{n}\right)$ converges to $f$ in $C^{1}\left(S^{1}, S^{1}\right)$. Since $f(x)=x$, there are proper closed intervals $K$ and $J$ on $S^{1}$ with $K \subset \operatorname{int}(J), f(K) \subset$ int $(J)$, and $x \in \operatorname{int}(K)$. For $n$ sufficiently large, the orbit of $x_{n}$ will be contained in $K$ and $f_{n}(K)$ will be contained in $J$. Hence, we can look at the restrictions of $f_{n}$ and $f$ to the interval $K$ and apply lemma 1 , as in the preceding paragraph, to show that $f^{\prime}(x)>0$ and $f^{\prime}(x) \leq-1$, a contradiction.

Proof of theorem 1. We have three cases.

Case 1 . $k$ is odd and $k \geq 3$. If $k=3$ the conclusion follows from lemma 2. Proceeding by induction, we assume the conclusion is true for all odd numbers less than $k$. By lemma $2, x$ is a periodic point of $f$ of period $r$ where $1<r \leq k$ and $k$ is a multiple of $r$. Hence, $k=r \cdot s$ where $s$ is an odd positive integer.

Let $g_{n}=\left(f_{n}\right)^{r}$ and $g=f^{r}$. Then $\left(g_{n}\right)$ converges to $g$ and $x_{n}$ is a periodic point of $g_{n}$ of period $s$ (for each $n$ ), but $x$ is a fixed point of $g$. By our induction hypothesis, $s=1$ and $k=r$.

Case 2. $k=2^{s}$ for some integer $s \geq 0$. If $k=1$ or $k=2$ the conclusion is immediate, so we assume $k \geq 4$. Let $g_{n}=\left(f_{n}\right)^{\left(\frac{1}{4} k\right)}$. Then for each $n, x_{n}$ is a periodic point of $g_{n}$ of period 4. By lemma $2, x$ is a periodic point of $g$ of period 2 or 4 . Hence, $x$ is a periodic point of $f$ of period $k$ or $\frac{1}{2} k$.

Case 3. $k=m \cdot r$ where $r=2^{s}$ for some $s \geq 1$ and $m$ is odd with $m \geq 3$. Note that the sequence $\left(f_{n}\right)^{r}$ converges to $f^{r}$ and (for each $n$ ) $x_{n}$ is a periodic point of $\left(f_{n}\right)^{r}$ of period $m$. Hence, by case $1, x$ is a periodic point of $f^{r}$ of period $m$. 
On the other hand, the sequence $\left(f_{n}\right)^{m}$ converges to $f^{m}$, so $x$ is a periodic point of $f^{m}$ of period $r$ or $\frac{1}{2} r$ by case 2 .

Let $t$ denote the period of $x$ as a periodic point of $f$. Then $\frac{1}{2} r$ and $m$ are relatively prime, $\frac{1}{2} r$ and $m$ divide $t$, and $t$ divides $k$. Hence, $t=k$ or $t=\frac{1}{2} k$ and the proof of theorem 1 is complete.

\section{Proof of theorem 2}

We need the follo $w^{\prime i n g ~ l e m m a ~ f o r ~ t h e ~ p r o o f ~ o f ~ t h e o r e m ~} 2$.

LEMMA 3. Let $f \in C^{1}(I, I)$ have a periodic orbit $\left\{x_{1}, x_{2}, \ldots, x_{n}\right\}$, where $x_{i}<x_{i+1}$ and $4 \leq n=2^{k}$, for some $k$. Suppose this orbit is 'separated to first order' [10] i.e. if $f\left(x_{i}\right)=x_{j}$ then $i \leq \frac{1}{2} n$ if and only if $\frac{1}{2} n<j$. Then there are points $y$ and $z$ in the interval $\left[x_{1}, f\left(x_{1}\right)\right]$ with $f^{\prime}(y) \leq-1$ and $f^{\prime}(z) \geq 0$. (The same statement holds for $\left[f\left(x_{n}\right), x_{n}\right]$ ). Proof. Let $j=\frac{1}{2} n$. Then $f\left(x_{j}\right) \geq x_{j+1}$ and $f\left(x_{j+1}\right) \leq x_{j}$, by hypothesis, so $\exists y \in\left[x_{j}, x_{j+1}\right] \subseteq$ $\left[x_{1}, f\left(x_{1}\right)\right]$ such that $f^{\prime}(y) \leq-1$. Next let $f\left(x_{i}\right)=x_{n}$, so $1 \leq i \leq j$. If $i=1$ the desired $z$ exists by lemma 1 . If $i>1$, then $f\left(x_{i-1}\right)<f\left(x_{i}\right)$ implies such a point exists in $\left[x_{i-1}, x_{i}\right] \subseteq\left[x_{1}, f\left(x_{1}\right)\right]$. (The final remark follows by taking the 'mirror image' $g(x)=1-f(1-x)$.)

Proof of theorem 2. We first consider the interval case, $f \in C^{1}(I, I)$. Since the set of periodic points is closed, every period is a power of $2([4],[11])$. Therefore, [3], any periodic orbit of $f^{k}$ is separated to order one under $f^{k}$, for any $k \geq 0$ (in [3] this is called 'simple').

Suppose now that all powers of 2 are periods of periodic points of $f$. Let $p_{n}$ be the least point in an orbit of period $2^{n}$, for each $n$. A subsequence of these must converge, say to $p$. Since the periodic points are a closed set, $p$ is periodic of some period $s$ (a power of 2). Let $g=f^{s}$, so $g(p)=p$, and note that for all $n, p_{n}$ is an endpoint of a periodic orbit of $g$ (separated to order one under $g$ ). Moreover, a subsequence of $\left\{g_{n}\left(p_{n}\right)\right\}$ converges to $g(p)=p$, but then (since $g \in C^{1}(I, I)$ ) lemma 3 implies that $g^{\prime}(p) \leq-1$ and $g^{\prime}(p) \geq 0$. Hence not all powers of 2 can be periods if the set of periodic points is closed, and so by the theorem of Sarkovskii, $f$ has but finitely many periods.

We next take up maps of the circle, $f \in C\left(S^{1}, S^{1}\right)$. We may assume $f$ has a fixed point (the set of periodic points of $f$ and of $f^{k}$ coincide). Were some period of $f$ not a power of $2, f$ would have positive topological entropy [5], and the set of periodic points would therefore not be closed [9]. Hence every periodic point of $f$ has a power of 2 as its period.

Choose an orientation of $S^{1}$, and let $\left\{p_{1}, \ldots, p_{k}\right\}$ be a periodic orbit, labelled in consecutive order so there are no points of that orbit interior to any of the intervals $M_{1}=\left[p_{1}, p_{2}\right], M_{2}=\left[p_{2}, p_{3}\right], \ldots, M_{k}=\left[p_{k}, p_{1}\right]$. By theorem $A_{1}$ of [2], some $M_{j}$ is not $f$-covered by any $M_{i}$ for $i \neq j$, i.e. $M_{j} \neq f(L)$ for any interval $L \subseteq M_{i}$. By renumbering, we may assume $j=k$. Denote by $I$ the complement (in $S^{1}$ ) of the interior of $M_{k}$. Then as in theorem $A_{2}$ of [2], $f$ restricted to $I$ is differentiably 'conjugate' to a map $g: I \rightarrow \mathbb{R}$. We may consider $\left\{p_{1}, \ldots, p_{m}\right\}$ to be a periodic orbit of $g$; it is separated to order one, and the 'endpoint' is well defined, so lemma 3 produces 
$y, z$ between $p_{1}$ and $f\left(p_{1}\right)$ with $f^{\prime}(y) \leq-1, f^{\prime}(z) \geq 0$. Proceeding as above, $f$ has finitely many periods.

\section{Proof of theorem 3}

We conclude by proving theorem 3 . We will use a theorem of [1], which states that if $f \in C^{0}(I, I)$ and $f$ has a point of period $n$, then there is a neighbourhood $N(f)$ in $C^{0}(I, I)$ such that for all $g \in N(f)$ and all $k$ with $k \triangleleft n, g$ has a periodic point of period $k$.

Proof. Let $t_{k}=\inf \left\{s \in[0,1]: f_{s} \in F(k)\right\}$. Note that this set is non-empty by the theorem of Sarkovskii, since $n \triangleleft k \triangleleft m$.

First suppose that $k$ is not a power of 2 . Let $s_{k}=t_{k}$. By corollary 1 (or if $k=\infty$ by corollary 2$), f_{s_{k}} \in F(k)$, so by the theorem of $[1], f_{s_{k}} \in G(k)$.

Now suppose that $k=2^{i}$ where $j \geq 1$. Let

$$
r=t_{2 k}=\inf \left\{s \in[0,1]: f_{s} \in F(2 k)\right\} \text {. }
$$

Then $f_{r} \notin F(4 k)$ (by the theorem of [1]), but $f_{r} \in F(k)$ (by corollary 2). If $f_{r} \in F(k) \backslash F(2)$ we let $s_{k}=r$. If $f_{r} \in F(2 k)$ we choose $\varepsilon>0$ sufficiently small that $f_{r-\varepsilon} \in F(k)$ (by the theorem of $[1]$ ), and let $s_{k}=r-\varepsilon$. In either case $f_{s_{k}} \in G(k)$.

\section{REFERENCES}

[1] L. Block. Stability of periodic orbits in the theorem of Sarkovskii. Proc. Amer. Math. Soc. 81 (1981), 333-336.

[2] L. Block. Periods of periodic points of maps of the circle which have a fixed point. Proc. Amer. Math. Soc. 82 (1981), 481-486.

[3] L. Block. Simple periodic orbits of mappings of the interval. Trans. Amer. Math. Soc. 254 (1979), 391-398.

[4] L. Block. Homoclinic points of mappings of the interval. Proc. Amer. Math. Soc. 72 (1978), 576-580.

[5] L. Block, J. Guckenheimer, M. Misiurewicz, \& L.-S. Young. Periodic points and topological entropy of one-dimensional maps. Lecture Notes in Math. No. 819, Springer: Berlin, 1980, pp. 18-34.

[6] P. Collet \& J. P. Eckmann. Iterated maps on the interval as dynamical systems. Progr. in Phys. Phys. 1 (Birkhauser, 1980).

[7] M. Feigenbaum. Quantitative universality for a class of non-linear transformations. J. Stat. Phys. 19 (1978), 25-52.

[8] M. Misiurewicz. Structure of mappings of an interval with zero entropy. Publ. Math. I.H.E.S., to appear.

[9] M. Misiurewicz. Horseshoes for mappings of the interval. Bull. Acad. Polon. Sci. 27 (1979), 167-169.

[10] Z. Nitecki. Topological dynamics on the interval. Ergodic Theory and Dynamical Systems, Vol. II (Proceedings of the Special Year, Maryland, 1979-1980) Progr. in Math. Birkhäuser, 1981, to appear.

[11] Z. Nitecki. Maps of the interval with closed period set. Proc. Amer. Math. Soc. 85 (1982) 451-456.

[12] A. Sarkovskii. Coexistence of cycles of a continuous map of the line into itself. Ukr. Mat. Z. 16 (1964), 61-71. (In Russian.) 